DOI 10.1007/s10773-009-9966-4)

\title{
ENTROPY OF THE UNIVERSE
} \section{(to be published by Internation Journal of Theoretical Physics}

\author{
Marcelo Samuel Berman ${ }^{1}$ \\ ${ }^{1}$ Instituto Albert Einstein / Latinamerica - Av. Candido Hartmann, 575 - \# 17 and \\ 80730-440 - Curitiba - PR - Brazil email: msberman@institutoalberteinstein.org
}

(Dated: (Original: circa 2002; Last version: $8^{\text {th }}$ December, 2008))

\begin{abstract}
After a discussion on several limiting cases where General Relativity turns into less sophisticated theories, we find that in the correct thermodynamical and cosmological weak field limit of Einstein's field equations the entropy of the Universe is $R^{3 / 2}$ - dependent, where $R$ stands for the radius of the causally related Universe. Thus, entropy grows in the Universe, contrary to Standard Cosmology prediction.
\end{abstract}




\title{
ENTROPY OF THE UNIVERSE
}

\author{
(to be published by Internation Journal of Theoretical Physics
}

DOI 10.1007/s10773-009-9966-4)

Marcelo Samuel Berman

\section{Introduction}

There are many different ways in order to compare General Relativity with Newtonian Theory. In the next Section, we shall introduce a cosmological weak field limit. In such limit, we can not ignore the existence of cosmic pressure and a cosmological "constant" term. The possibility of introducing cosmic pressure was surligned by Peacock (1999). Earlier, Ray d'Inverno (1992) had worked with a cosmological repulsive acceleration in Newtonian Cosmology. I recall Peter Landsberg and Evans (1977) having done something similar.

Other limits are possible, like the Machian (Berman, 2008a). A linearized theory of the electromagnetic type, was also devised as some kind of Sciama's limit to the field equations (Sciama, 1953; Berman, 2008b).

Barrow (1988), has worked what we shall call the thermodynamical Newtonian "limit". He established the two energy conservation equations, namely, the Newtonian and the thermodynamical; the latter implies the following definition of pressure,

$$
p=-\frac{d M}{d V} \quad,
$$

where $M$ and $V$ represent the mass and volume of a given system. He showed that both equations led to Robertson-Walker's field equations of General Relativity, provided that the energy density term, instead of being related to mass energy, should be related to all forms of energy, like, for instance, radiation.

As to the Machian limits, Berman (2008a; 2007) has shown that there are NewtonianMachian and General Relativistic Machian limits, which, in fact, result in the same conditions for the relevant Physical quantities. 
We must deny, that the strong but wrong impression in the air, surrounding Newtonian Cosmology as a pressureless model, is, in fact, true (Barrow, 1988).

\section{Cosmological Newtonian limit of field equations}

Standard Cosmology (Weinberg, 2008), introduces constant entropy. We shall show in next Section, that the Universe, could bear a growing entropy, in the correct limit of Einstein's field equations.

It is well known that Einstein's field equations, in the so-called Newtonian limit, reduce to Poisson's equation,

$$
\nabla^{2} \Phi=4 \pi G \sigma
$$

where $\Phi, G$ and $\sigma$ stand for potential, gravitational constant and gravitational energy density. What probably never was told, is that $\sigma$ represents not only the effective energy density, but also eventual pressure and cosmological "constant" terms. In fact, when applied in large scale, a pressure term may appear in the system, and in a cosmological scale, a lambda term is possible. In Whitrow's paper (Whitrow, 1946; Whitrow and Randall, 1951 ), he equated the inertial energy of the Universe $\left(M c^{2}\right)$ to the gravitational potential energy $\left(G \frac{M^{2}}{R}\right)$, finding the approximate relation $G \frac{M}{R} \cong c^{2}$.

If we postulate sphericity (the Universe resembles a "ball" of approximate spherical shape), egocentrism (each observer sees the Universe from its center) and democracy (each point in space is equivalent to any other one - all observers are equivalent), we may write, for each observer, the following Newtonian potential,

$$
\Phi=-G \frac{M}{R}
$$

In the needed interpretation, we shall see $R$ as the radius of the causally related Universe.

Then, from (1) and (2), we find,

$$
\nabla^{2} \Phi=\frac{\partial^{2} \Phi}{\partial R^{2}}=-2 \pi G \frac{M}{R^{3}}=4 \pi G\left(\rho+3 \frac{p}{c^{2}}-\frac{\Lambda}{4 \pi G}\right)
$$

The obvious solution remains,

$$
\rho=\rho_{0} R^{-2}
$$




$$
\begin{aligned}
& p=p_{0} R^{-2} \\
& \Lambda=\Lambda_{0} R^{-2}
\end{aligned}
$$

In the above, $\rho_{0} \quad, \quad p_{0}$ and $\Lambda_{0}$ are constants. The resulting equation is, for baryonic matter,

$$
M=\gamma R \equiv-2\left[\rho_{0}+3 \frac{p_{0}}{c^{2}}-\frac{\Lambda_{0}}{4 \pi G}\right] R>0
$$

We show that negative pressures are possible. As dark matter is represented by a positive cosmological term, for an accelerating Universe, we have $\Lambda_{0}>0$. On the other hand, the weak energy condition, stated as the positivity of energy density, should also apply and, thus, $\rho_{0}>0$. From (5), we find that $p_{0}<\frac{\Lambda_{0} c^{2}}{12 \pi G}-\frac{1}{3} \rho_{0} c^{2}$.

We can check that Whitrow's relation is retrieved in its generality.

\section{Speaking of Thermodynamics}

We saw above, that energy densities are $R^{-2}$ - dependent. If all energy densities are as such, we can say that the radiation component $\rho_{\text {rad }}$ has the same dependence, to wit,

$$
\rho_{\text {rad }}=b R^{-2}=a T^{4}
$$

In the above, $a$ and $b$ are constants while $T$ stands for absolute temperature, and the right hand side represents black-body radiation. We find the same relation that Kolb and Turner (1990) have already mentioned in another context, namely,

$$
T^{2} R=\text { constant }
$$

In this case, the entropy of such Universe is given by (Sears and Salinger, 1975),

$$
S=S_{0} R^{3 / 2} \quad\left(S_{0}=\text { constant }\right) \quad .
$$

This entropy is growing, because $\dot{R}>0$ for expanding Universes. Weinberg (1972), suggested that there would be dissipative processes in the Universe, that could be represented by viscosity terms.

\section{Final considerations}


Some comments on the above Sections are necessary.

first) As a by-product of our presentation, we can check that there is no infinite singularity in the beginning of this Universe, i.e., according to (5),

$$
\lim _{R \longrightarrow 0} M \longrightarrow 0
$$

second) We have also found that pressure and density obey a perfect gas equation of

state, for both are $R^{-2}$ - dependent. As dark-energy is representable by the lambda-term, this has also the same dependence, which is consistent with Modern Cosmology (Weinberg, 2008).

third) The thermodynamical conclusion of ours, is also confirmed by prior work on the above subjects, which was done by Berman (2007; 2007a; 2007b; 2008).

\section{Acknowledgements}

First of all, I need to stress the importance of an anonymous referee's report, whose comments directed me to write this version of the paper. I thank Nelson Suga, Marcelo F. Guimarães, Antonio F. da F. Teixeira, and Mauro Tonasse; I am also grateful for the encouragement by Albert, Paula, and Geni. I offer this paper in memoriam of M. M. Som

\section{References}

Barrow, J.D. (1988) - The Inflationary Universe, R. Blin Stoyle \& W.D. Hamilton (eds), Adam Hilger, Bristol. Paper presented at the University of Sussex, date $7-9^{\text {th }}$ September, 1987, on Interactions and Structures in Nuclei.

Berman,M.S. (2007) - The Pioneer Anomaly and a Machian Universe, Astrophysics and Space Science, 312, 275.

Berman,M.S. (2007a) - Introduction to General Relativity and the Cosmological Constant Problem, Nova Science, New York.

Berman,M.S. (2007b) - Introduction to General Relativistic and Scalar-Tensor Cosmologies, Nova Science, New York. 
Berman,M.S. (2008) - A Primer in Black-Holes, Mach's Principle, and Gravitational Energy , Nova Science, New York.

Berman,M.S. (2008a) - General Relativistic Machian Universe, Astrophysics and Space Science, 318, 273-277.

Berman,M.S. (2008b) - On the Machian Origin of Inertia, Astrophysics and Space Science, 318, 269-272.

d'Inverno, R. (1992) - Introducing Einstein's Relativity, Clarendon Press, Oxford.

Kolb, E.W.; Turner, M.S. (1990) - The Early Universe, Addison-Wesley, N.Y.

Landsberg, P. T.; Evans, D.A. (1977) - Mathematical Cosmology, Oxford Universe Press, Oxford.

Peacock, J.A. (1999) - Cosmological Physics, Cambridge Universe Press, Cambridge, page 25 , formula (1.85).

Sciama, D.N. (1953) - MNRAS, 113, 34.

Sears, F.W.; Salinger,G.L. (1975) - Thermodynamics, Kinetic theory, and Statistical Thermodynamics, Addison-Wesley, New York.

Weinberg, S. (1972) - Gravitation and Cosmology, Wiley, New York.

Weinberg, S. (2008) - Cosmology, Oxford University Press, Oxford.

Whitrow, G. (1946) - Nature, 158, 165.

Whitrow, G.; Randall, D. (1951) - MNRAS, 111, 455. 